\title{
EDITORIAL
}

\section{El liderazgo, la cultura y los desafíos de la educación ${ }^{1}$}

Las organizaciones en la actualidad se encuentran en un entorno cada vez más complejo y más cambiante. Los problemas a los que se enfrentan son más difíciles de comprender y de estimar alguna solución óptima. Si bien se cuenta con más recursos, mejores tecnologías y mayores comodidades que antaño, resulta más difícil describir los problemas, explicar las relaciones entre las variables y predecir su comportamiento. Las organizaciones, en su propósito, necesitan enfrentar esta complejidad y cambio, adaptándose permanentemente. En esta lucha por la supervivencia, han dejado de producir certezas a los colaboradores y hoy más que antes ofrecen incertidumbres.

La toma de decisiones es un proceso crítico y de alta sensibilidad en ambientes de incertidumbre ya que sus efectos son más significativos para la supervivencia de las organizaciones. Las decisiones exitosas son cada vez menos el producto de una mente brillante y cada vez más el resultado de una interacción armoniosa entre personas de diferentes disciplinas. En ese proceso, el liderazgo, definido como el arte de solucionar problemas relevantes, demuestra ser un factor determinante en la eficacia de las organizaciones porque, según una adecuada aplicación de estilos, puede asegurar una estrategia de mayor calidad. Es por esta razón que la toma de decisiones requiere de un liderazgo efectivo y de una adecuada aplicación de estilos.

En la aplicación de diferentes estilos de liderazgo es importante la composición del equipo directivo, siendo la heterogeneidad cognitiva y la homogeneidad valórica claves para la efectividad de la toma de decisiones. Estas condiciones relevan la cultura en la efectividad del liderazgo, entendiéndose como el conjunto de prácticas, valores y creencias compartidas entre los miembros de una organización. Las formas de relacionarse, las priorizaciones y las representaciones de la realidad pueden ser diferentes en grupos interdisciplinarios y especialmente, multiculturales.

La confianza es clave para que un equipo directivo tome buenas decisiones. Cuando los miembros de un equipo confían entre sí, a pesar de su heterogeneidad cognitiva, se atreven a divergir y piensan críticamente, surgiendo conflictos que, si se gestionan en forma adecuada, mediante debates con fundamentos técnicos, producen un mayor compromiso en aquellos acuerdos alcanzados, mayor responsabilidad en la rendición de cuenta y mejores resultados en la solución del problema. Si es baja la confianza, los miembros del equipo tienden a estar de acuerdo en casi todo y en aquello que no, consensuan, decidiendo por lo que no genera conflictos y produciendo bajos niveles de compromiso, escasa responsabilidad y resultados deficientes en la solución de los problemas.

Sociedades con alta evitación de incertidumbre, alta distancia de poder y bajo individualismo se caracterizan principalmente por decisiones poco innovadoras y fuertemente influenciadas por una autoridad y por un colectivo. La evitación de incertidumbre se entiende como todo aquello que una sociedad hace para controlar lo incontrolable, la distancia de poder, como la resignación que tienen las personas ante las diferencias de poder que existen en los grupos o en la sociedad y el colectivismo como la mayor relevancia que tiene el colectivo sobre las decisiones de los individuos².

\footnotetext{
1 Los autores agradecen al proyecto FONDECYT 1170960 denominado "La calidad en las instituciones de educación superior: la influencia de los estilos de liderazgo y la cultura académica".

2 G. HOFSTEDE, J. Hofstede, J. y M. Minkov. "Cultures and Organizations: Software of the Mind". McGraw-Hill. 3ra. Edición, pp. 90- 102. NY, EEUU. ISBN: 978-0-07-177015-6. Año 2010.
} 
Países miembros de la Organización para la Cooperación y el Desarrollo Económicos (OCDE) como Noruega, Suiza, Alemania, Dinamarca, Holanda, Suecia, Canadá, Bélgica, Luxemburgo y Francia se caracterizan en promedio por índices de evitación de incertidumbre (58\%), distancia de poder (40\%) y colectivismo (28\%) relativamente bajos. Estos países se encuentran actualmente dentro de las veinticinco mejores ubicaciones en los índices de competitividad global ${ }^{3}$, desarrollo humano ${ }^{4}$ y equidad $\mathrm{OCDE}^{5}$. Estos diez países que, desde este enfoque, son los más ejemplares de la OCDE se caracterizan por ser sociedades horizontales e individualistas.

En este sentido merece la pena indicar que Chile, país miembro de la OCDE desde el año 2010, se encuentra en puestos de muy alto nivel en los índices de competitividad (35) y de desarrollo humano (38) sin embargo es unos de los países de menor equidad en el mundo. La sociedad chilena se caracteriza por índices de evitación de incertidumbre (86\%), distancia de poder (63\%) y colectivismo (77\%) muy altos, siendo catalogada como una sociedad vertical y colectivista.

La sociedad chilena es culturalmente bastante diferente a este grupo de los países más ejemplares de la OCDE y del mundo. Aunque, tiene una mayor cercanía cultural con Bélgica y Francia, países que se caracterizan por índices de evitación de incertidumbre (Bel 94\% y Fran 86\%) y de distancia de poder (Bel $65 \%$ y Fran 68\%) altos. Sin embargo, tiene una importante brecha en el índice de colectivismo (Bel 25\%, Fran 29\% y Chi 77\%). Bélgica y Francia son sociedades tan individualistas como el resto de los países que forman parte de este grupo selecto. Es posible que los altos índices de inequidad que caracterizan a la sociedad chilena se expliquen desde su alto índice de colectivismo ${ }^{6}$.

El colectivismo podría ser una dimensión cultural que inhiba el comportamiento autónomo y la libertad de acción de las personas al interior de los grupos, y de la sociedad en general, por una lealtad y compromiso mal entendidos. El colectivismo enfatiza las relaciones de parentesco y la conformidad y armonía de pensamiento, sentimientos y acción. Los miembros de un equipo directivo se pueden ver influenciados a no contradecir al grupo o a personas dominantes en éste por temor al conflicto y a la vulnerabilidad que esto le pudiera significar. El pensamiento crítico y la motivación al logro pueden ser herramientas que ayuden a las personas a colaborar en un colectivo sin perder de vista sus objetivos personales, su autonomía y su privacidad.

Ahora bien, la educación en Chile y en Latinoamérica en general se caracteriza por favorecer especialmente las habilidades técnicas y de conceptualización, sin realizar importantes esfuerzos en la formación de liderazgo o también llamadas habilidades sociales. Se estima relevante que la educación asuma el objetivo de formar la autonomía de las personas, pero al mismo tiempo el respeto a la individualidad y la colaboración a los colectivos. La autonomía, el respeto y la colaboración han de ser valores fundamentales de la formación de las personas en Chile, toda vez que en su conjunto pueden favorecer su liderazgo al interior de un equipo directivo o de cualquier colectivo.

En definitiva, contar con integrantes de un equipo o de una colectividad capaces de ejercer liderazgo con autonomía, respeto y colaboración, más allá de las herramientas conceptuales y técnicas necesarias, podría hacer posible que las personas y las organizaciones avancen con mejores resultados en la solución de sus problemas relevantes. Así también, porque no estimarlo, que nuestra sociedad avance en la solución

\footnotetext{
3 WORLD ECONOMIC FORUM. "Global Competitiveness Report 2015-2016”. 1st September 2015. Obtenido el 11 de septiembre de 2018 en http://reports.weforum.org/global-competitiveness-report-2015-2016/competitiveness-rankings/

4 PROGRAMA DE NACIONES UNIDAS PARA EL DESARROLLO (2016). "Informe sobre Desarrollo Humano 2016". 2016. Pp. 22- 25 Obtenido el 11 de septiembre de 2018 en http://hdr.undp.org/sites/default/files/HDR2016_SP_Overview_Web.pdf

5 ORGANIZACIÓN PARA LA COOPERACIÓN Y EL DESARROLLO ECONÓMICOS. "Gini Coefficient, 2015 ". 2015. Obtenido el 11 de septiembre de 2018 en http://www.oecd.org/social/inequality.htm

6 HOFSTEDE INSIGHT. Obtained on September 11, 2018 at https://www.hofstede-insights.com/country-comparison/ belgium,chile,france/
} 
de la inequidad que nos impide alcanzar el objetivo de ser una sociedad justa, prospera y desarrollada. La educación necesita asumir el desafío del liderazgo y la transformación cultural de nuestra sociedad.

\author{
Dr. Jaime Riquelme-Castañeda \\ Universidad Finis Terrae \\ Centro de Liderazgo \\ Santiago, Chile \\ E-mail: jriquelme@uft.cl
}

\author{
Dra. Liliana Pedraja-Rejas \\ Universidad de Tarapacá \\ Ingeniería Industrial \\ Arica, Chile \\ E-mail: lpedraja@uta.cl
}

